\title{
Clinical Laboratorial Perspective on Screening of Prediabetes and need for Standardization of Post Prandial Blood Glucose Testing in Nepal
}

\author{
Dr. Vivek Pant ${ }^{1}$, Dr. Santosh Pradhan ${ }^{1}$, Dr. Keyoor Gautam ${ }^{2}$ \\ ${ }^{1}$ Department of Clinical Biochemistry, Samyak Diagnostic Pvt. Ltd, Lalitpur, Kathmandu, Nepal \\ ${ }^{2}$ Department of Pathology, Samyak Diagnostic Pvt. Ltd, Lalitpur, Kathmandu, Nepal
}

\begin{abstract}
This study explores the differences in categorization of prediabetes based on fasting blood glucose by the World Health Organization and the American Diabetes Association from a laboratory perspective and the associated implications. As the diagnostic thresholds for prediabetes are based on the likelihood of developing overt diabetes, the criteria for this collective representation of dysglycemic states, including Impaired Fasting Glucose and Impaired Glucose Tolerance needs to be clearly established in the face of a society that grows increasingly concerned about diabetes and its associated complications over time. The authors intend to delve into different published points of view and recommend a categorization in Nepal's context.
\end{abstract}

Keywords: fasting blood glucose; post-prandial blood glucose; prediabetes; 75 grams oral glucose tolerance test

\section{Introduction:}

Existing norms of diagnosing prediabetes are based on measurements of glycated hemoglobin (HbA1c), fasting blood glucose or blood glucose concentration two hours following a 75 grams oral glucose load. The term prediabetes has been accepted to represent either of two states, the first where an individual cannot maintain baseline blood sugar levels over periods of fasting (at least eight $\left[\mathrm{r}^{1}\right]$ hours), thus aptly labeled Impaired Fasting Glucose (IFG) and / or a second state where an individual cannot handle glucose loads as efficiently, indicated by the term, Impaired Glucose Tolerance (IGT). The American Diabetes Association (ADA) and the World Health Organization (WHO) have defined different thresholds for the categorization for prediabetes. According to the ADA, a fasting blood glucose level of at least $100 \mathrm{mg} / \mathrm{dL}(5.6$

Correspondence Author

Dr. Vivek Pant, MD Clinical Biochemistry

Samyak Diagnostic Pvt. Ltd, Lalitpur, Kathmandu, Nepal

Email: drvpant@gmail.com

Phone no.: +977 9841486789 $\mathrm{mmol} / \mathrm{L})$ but less than $126 \mathrm{mg} / \mathrm{dL}(7.0 \mathrm{mmol} / \mathrm{L})$ is categorized as IFG, while a blood glucose level in between $140 \mathrm{mg} / \mathrm{dL}(7.8 \mathrm{mmol} / \mathrm{L})$ and $200 \mathrm{mg} / \mathrm{dl}$ (11.1 mmol/L) 2 hours following an oral glucose load of 75 -grams qualifies as IGT. ${ }^{1}$ The WHO, however, defines IFG as a state where fasting blood glucose levels are between $110 \mathrm{mg} / \mathrm{dl}(6.1 \mathrm{mmol} / \mathrm{L})$ and $126 \mathrm{mg} / \mathrm{dL}(7.0 \mathrm{mmol} / \mathrm{L})$, whereas the WHO's definition for IGT uses the same thresholds as that of the ADA. ${ }^{2}$ In this regard, a person with a fasting blood sugar level of $105 \mathrm{mg} / \mathrm{dl}$ would be regarded as prediabetic by ADA standards, while he would be dismissed as normal when using the WHO definitions. It is interesting to note that the ADA also uses $\mathrm{HbAlc}$ values ranging from $5.7 \%$ to $6.4 \%$ in the categorization of pre-diabetes, regardless of blood sugar values.

The progression from prediabetes to overt hyperglycemic symptoms of type 2 diabetes arguably occurs over a period of many years. ${ }^{3}$ The likelihood of progression to overt diabetes is significantly more in patients with both IFG 
and IGT, as compared to patients with a single impairment of either IFG or IGT. ${ }^{4}$ Insulin resistance and other lifestyle related factors also contribute to an elevation of this risk in susceptible individuals.

\section{Controversies:}

To start with, the term prediabetes can be misleading - because many people diagnosed with IFG or IGT do not ever develop overt diabetes. Up to $70 \%$ of prediabetic, figures have shown, will develop diabetes at some point in their lifetime. ${ }^{5}$ In contrast to a normoglycemic state, this prediabetic state has been established to be associated with an increased occurrence of micro- and macrovascular complications - when long term glycemic control is taken into account -regardless of whether an individual progresses to overt type 2 diabetes. ${ }^{6}$ The importance of screening for prediabetes cannot be overemphasized here, given that Nepal has been witnessing an alarming rise in the number of diabetes cases over the past decade along with a population that grows increasingly concerned with the complications related to diabetes.

The WHO has recommended using the term intermediate hyperglycemia to indicate glycemic levels varying between normal glucose tolerance and overt diabetes. ${ }^{2}$ The cut-offs defined by ADA and WHO for establishing prediabetes do not equate, owing to the fact that the reference levels for prediabetes were most likely established by these organizations using different groups of individuals with their unique lifestyle differences and ethnic origins. The primary concern here is that these threshold values should be sensitive enough to correctly identify individuals at an increased risk. It is blatantly clear that using the ADA recommendations, a larger fraction of the population would be diagnosed as prediabetic as compared to when using the WHO cut-offs. In a meta-analysis published in $2017,27 \%$ of the population studied was identified as pre-diabetics when WHO guidelines were used, compared to a whopping $48 \%$ if ADA criteria were to be used in the same population. ${ }^{7}$ It is interesting to note that the substitution of the ADA criteria in place of the WHO criteria for the screening and diagnosis of type 2 diabetes thus potentially doubles the estimated prevalence of prediabetes. It should be noted that this labeling people as prediabetes has implications which include periodic and frequent blood testing, a social stigma associated with the diagnosis, some forced lifestyle changes and the use of certain medications.

To put it bluntly, the usefulness of IFG and IGT in evaluating glycemic control has long been criticized due to the variability in threshold values and in their prognostic worth in reference to probability of developing diabetes or the likelihood and severity of related complications. ${ }^{8}$ Also, these threshold values for diagnosis of prediabetes are limited in application - owing to poor reproducibility. ${ }^{9}$ Nevertheless, the presence of IFG and / or IGT in an individual identifies him or her with having different pathological abnormalities in their glucose homeostasis which needs to be addressed; there is no argument to that.

\section{Nepalese Context:}

The prevalence of type 2 diabetes reported in a recent study in Nepal is $8.5 \%{ }^{10}$ Similarly, the prevalence of prediabetes in a community-based study done in Nepal, is reported to be $7.14 \% .{ }^{11}$ In our experience, prediabetes is often an incidental diagnosis in people who undergo a routine physical workup or perhaps a screening for diabetes.

Most of the clinical laboratories in Nepal use an established reference range for fasting and postprandial blood glucose, as recommended by the WHO. The cut off value established by both the ADA and the WHO for post prandial blood glucose levels is based on the premise that the patient undergoing the test has consumed carbohydrates equivalent to 75 grams of an oral anhydrous glucose solution. Our practice of measuring post prandial blood sugar is via a blood sample taken two hours following lunch and this is based on the assumption that the major portion of our diet comprises carbohydrates. 
Given that the established reference ranges are based on the oral 75 grams anhydrous glucose load, it is quite valid to argue that in our settings, the established diagnostic sensitivity of post prandial blood glucose tests are greatly contended, at best. This emphasizes the need for standardization of post prandial blood glucose testing. Save for a few laboratories in Nepal that routinely perform the 75 grams oral glucose tolerance test in screening gestational diabetes, very few organizations have the concept of a standardized oral glucose load prior to evaluating post prandial glucose. Again, the fact that this concept of oral glucose loading has additional implications associated with it, because it is definitely more time consuming, may incite malingering and decrease compliance on part of the patients, owing to the hassle involved and there is the possibility that subsequent results may advocate additional confirmatory tests.

With the advent of $\mathrm{HbA} 1 \mathrm{c}$ as a standardized marker of long term glycemic control as well as of patient compliance, the ADA eventually advocated its use in the screening and diagnosis of prediabetes, based on widespread studies on reference intervals. The WHO, too has included the use of HbAlc in the diagnosis of diabetes recently. The catch is that we do not have enough evidence to justify using $\mathrm{HbA} 1 \mathrm{c}$ in the evaluation of prediabetes (IFG and/ or IGT states). Also, with the costs of laboratory estimation of $\mathrm{HbAlc}$ amounting to roughly ten times that of the cost of estimating blood sugar, it is a no-brainer that blood glucose investigation is the better option for Nepal, which still relies on a cash-based healthcare delivery system. It is also imperative that the ADA recommendations for fasting blood glucose thresholds be implemented in Nepal in the face of the growing number of cases of diabetes and related complications in the urban population.

\section{Recommendation:}

For patients that undergo a routine health workup or in patients that are screened for type- 2 diabetes, a fasting blood glucose measurement between 100 and $126 \mathrm{mg} / \mathrm{dL}$ on two or more occasions should advocate a standardized 75 grams oral anhydrous glucose (82.5 grams of monohydrate glucose available in Nepal) tolerance test to establish prediabetes in our context.

\section{Conclusion:}

Testing for blood glucose following $75 \mathrm{gm}$ oral glucose load using the diagnostic thresholds advocated by the ADA standardizes both the diagnostic testing for prediabetes, as well as the evaluation of long-term glycemic control in diabetic patients, in terms of reporting accuracy, value for money, as well as predictive values from a clinician's perspective.

\section{Acknowledgement:}

Author would like to acknowledge Dr. Aseem Bhattarai for language editing of this manuscript.

Conflict of Interest- None

\section{References:}

1. American Diabetes Association. Diagnosis and classification of diabetes mellitus. Diabetes care. 2014 Jan 1;37(Supplement 1):S81-90. https://doi.org/10.2337/dc14-S081

2. WHO, International Diabetes Federation. Definition and diagnosis of diabetes mellitus and intermediate hyperglycemia: report of a WHO/IDF consultation. 2006. Geneva: World Health Organization. http://apps.who.int/iris/ bitstream/10665/43588/1/9241594934_eng. pdf (accessed July 22, 2020).

3. Weyer C, Bogardus C, Mott DM, Pratley RE. The natural history of insulin secretory dysfunction and insulin resistance in the pathogenesis of type 2 diabetes mellitus. The Journal of clinical investigation. 1999 Sep 15;104(6):787-94. https://doi.org/10.1172/ jci7231

4. Gerstein HC, Santaguida P, Raina P, Morrison $\mathrm{KM}$, Balion C, Hunt D et al. Annual incidence and relative risk of diabetes in people with various categories of dysglycemia: a systematic overview and meta-analysis of prospective 
studies. Diabetes research and clinical practice. 2007 Dec 1;78(3):305-12. https://pubmed.ncbi. nlm.nih.gov/17601626/

5. PerreaultL, FærchK. Approaching pre-diabetes. Journal of Diabetes and its Complications. 2014 Mar 1;28(2):226-33. https://pubmed.ncbi. nlm.nih.gov/24342268/

6. Palladino R, Tabak AG, Khunti K, Valabhji $\mathrm{J}$, Majeed A, Millett $\mathrm{C}$ et al. Association between pre-diabetes and microvascular and macrovascular disease in newly diagnosed type 2 diabetes. BMJ Open Diabetes Research and Care. 2020 Apr 1;8(1):e001061. https://www. ncbi.nlm.nih.gov/pmc/articles/PMC7202749/

7. Barry E, Roberts S, Oke J, Vijayaraghavan $\mathrm{S}$, Normansell R, Greenhalgh T. Efficacy and effectiveness of screen and treat policies in prevention of type 2 diabetes: systematic review and meta-analysis of screening tests and interventions. bmj. 2017 Jan 4; 356:i6538. https://pubmed.ncbi.nlm.nih.gov/28052845/

8. Genuth S, Kahn R. A step backward-or is it forward? Diabetes Care. 2008 Jun 1; 31(6):1093-6. https://doi.org/10.2337/dc072407
9. Balion CM, Raina PS, Gerstein HC, Santaguida PL, Morrison KM, Booker L et al. Reproducibility of impaired glucose tolerance (IGT) and impaired fasting glucose (IFG) classification: a systematic review. Clinical Chemistry and Laboratory Medicine (CCLM). 2007 Sep 1;45(9):1180-5. https://pubmed.ncbi. nlm.nih.gov/17635074/

10. Dhimal M, Karki KB, Sharma SK, Aryal KK, Shrestha N, Poudyal A, Mahato NK, Karakheti A, Sijapati MJ, Khanal PR, Mehata S. Prevalence of selected chronic non-communicable diseases in Nepal. Journal of Nepal Health Research Council. 2019 Dec 13;17(3):394-401. https:// pubmed.ncbi.nlm.nih.gov/31735938/

11. Silvanus V, Dhakal N, Pokhrel A, Baral BK, Panta PP. Community based screening for diabetes and prediabetes using the Indian Diabetes Risk Score among adults in a semiurban area in Kathmandu, Nepal. Nepal Medical College Journal. 2019 Mar 31;21(1):12-20. https://doi.org/10.3126/nmcj.v21i1.24839 